\title{
Development of an STM32 Microprocessor System for Automatic Continuous Remote Temperature Control in an Intensive Flow of People
}

\author{
Ilgiz Bakiev
}

\begin{abstract}
This article discusses the fundamental principles of non-contact temperature measurement, technical solutions that translate them into practice in order to automate temperature control and improve the current epidemiological situation in the world in connection with coronavirus infection, as well as a proposal for a new technical solution that is more affordable, which will increase coverage of the use of automatic systems for monitoring the body temperature of people in public places, and a description of the following steps for its implementation.
\end{abstract}

Keywords - Coronavirus, Infrared sensor, Microcontroller, Non-contact temperature measurement.

\section{INTRODUCTION}

Temperature measurement is one of the most crucial stages of human cognition of the surrounding world. It lies at the bottom of measuring any physical parameters directly related to it. To date, contact thermometers, which underlie bringing into physical contact with an investigated body, achieving thermodynamic equilibrium, and determining changes in an intermediate physical parameter correlated with the desired temperature value, keep retaining their popularity.

Declared by WHO as a pandemic on March 11, 2020, SARS-CoV-2 coronavirus infection has had a catastrophic impact on the modern way of life. In order to reduce the virus spread among the healthy population by the infected people and accelerate the rate of the pandemic eradication, mandatory temperature control activities are carried out virtually throughout all public places of the world; they aim at identifying people with fever, which is one of the symptoms of the coronavirus infection [1], and preventing their prolonged presence in areas of mass crowds of people.

The use of contact thermometers implies waiting for a certain amount of time to establish temperature equilibrium and imposes their continuous treatment with antiseptic agents for hygienic purposes. In such cases, it is most expedient to use devices for non-contact temperature measurement with a short response time, also known as pyrometers, whose operation principle underlies measuring the intrinsic thermal radiation mainly in the infrared and visible light ranges emitted by an investigated body [2].

Submitted on September 14, 2021

Published on October 18, 2021.

Ilgiz Bakiev, Kazan National Research Technical University named after

A.N. Tupolev - KAI, Russia.

(e-mail: BakievII@stud.kai.ru)
However, widespread pyrometers found in local pharmacies require manual use, which increases the time for passing temperature control in a large flow of people, and, together with the negligence of the responsible persons, makes it perfunctory whatsoever. Automation of temperature control of large flows significantly increases its efficiency.

Automation of temperature control implies continuous registration of infrared radiation and their transmission to a processing device for further display of the processed information on output devices, for example, on a monitor screen placed directly in front of a responsible person, reacting appropriately in case of detection of a person with an elevated body temperature, meaning a possibility of their experience of fever.

\section{CASe Study}

If an object has a temperature higher than the absolute zero temperature, it radiates electromagnetic waves proportional to its temperature afterward. Part of the radiation sent is infrared radiation which contains information about the temperature. Infrared radiation emitted by an object passes through the environment and focuses on a detector element by a lens or input optics. Due to the radiation registration, the detector element generates an electrical signal proportional to it. The signal processing into an output quantity proportional to the object temperature is carried out through signal amplification, analog signal into a digital signal conversion, and subsequent digital processing. Later, the temperature value can be sent on the display or output as a signal.

The structure of a device that performs non-contact temperature measurement is shown in Fig. 1.

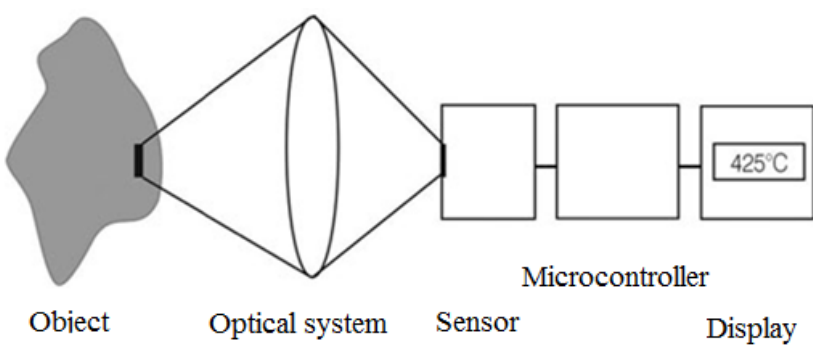

Fig. 1. Structure of a non-contact temperature measurement device.

The optical system is necessary for focusing the received radiation; it increases the device operating range while maintaining its low observational error. Moreover, the system contains an infrared filter that delays the 
electromagnetic radiation passing through it with the exception of radiation lying in the infrared wavelength range; the filter prevents extraneous radiation from entering the infrared sensor and, consequently, reduces measurement errors.

The infrared sensor operation underlies the pyroelectric effect that implies the appearance of an electric field in the crystals of pyroelectric materials due to a change in their temperature, which occurs in particular as a result of thermal radiation. When pyroelectrics are heated or cooled, they polarize in proportion to the change in temperature, resulting in a short-term electrical potential difference [3].

The detector output signal is related to the target object irradiance, which is directly proportional to the temperature of the emitted object, according to the Stefan-Boltzmann law, which has the following form [4]:

$$
j^{*}=\varepsilon \sigma \Delta T^{4}
$$

where

$j^{*}$ - irradiance, $W m^{-2}$;

$\varepsilon$ - emissivity;

$\sigma$ - Stefan-Boltzmann constant;

$\Delta T$ - temperature difference, $K$.

The output analog signal is converted into a digital signal using an analog-to-digital converter, then using the obtained value and mathematical operations, the temperature difference between the current and previous measurements is calculated:

$$
\Delta T=\sqrt[4]{\frac{j^{*}}{\varepsilon \sigma}}
$$

The calculated difference is compared with the previous temperature value, and thus a new value is obtained. The transformations described above can be done both using the microcontroller resources and by means of additional elements inside the sensor. In any case, the obtained temperature value is written into the microcontroller memory, which can then be transmitted to other devices.

A microcontroller is a single-chip computer made in the form of an integrated circuit that combines the functions of a processor, peripheral devices, such as ROM and RAM [5].

In addition to the method above, contactless temperature measurement is also permissible through determining the wavelength with the maximum intensity using Wien's displacement law, which establishes the dependence of the wavelength at which the spectral flux density of black body radiation reaches its maximum on the temperature of the black body, and is expressed by an equation [6]:

$$
\lambda_{\max }=\frac{b}{T}
$$

Here the coefficient $b$ combines several physical constants at once:

$$
b=\frac{c h}{k \alpha}
$$

where: $c$ - speed of light in vacuum;

$h$ - Planck's constant;

$k$ - Boltzmann constant;

$\alpha$ - Wien's constant.

Wien's displacement law is graphically illustrated in Fig. 2.

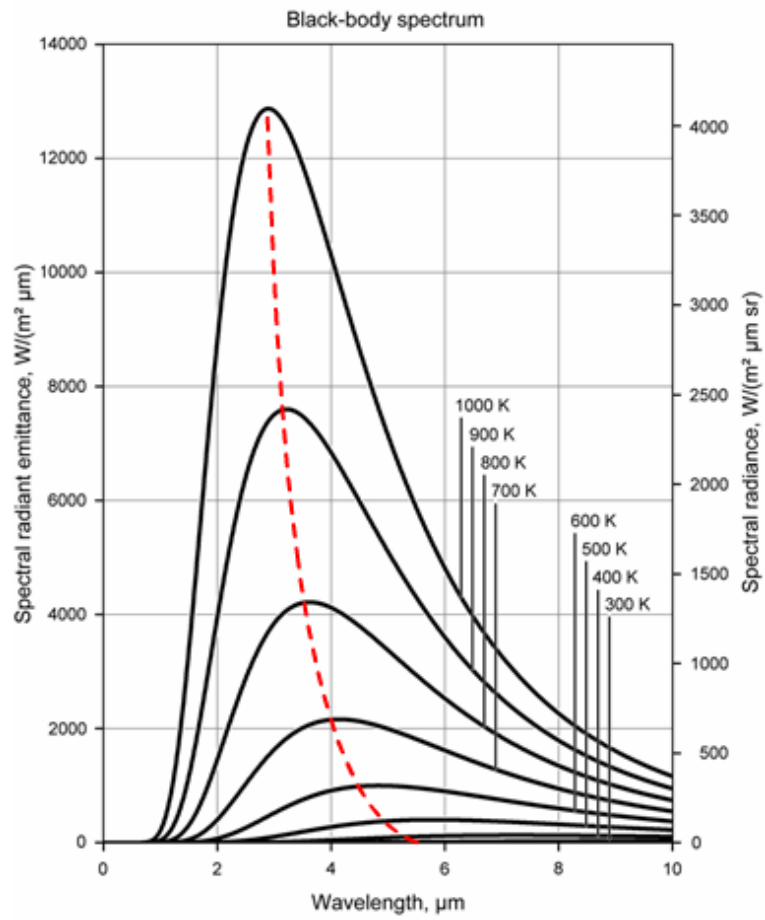

Fig. 2. Wien's displacement law.

Emissivity is the ratio of the radiation energy of a given body to the radiation energy of a black body with the same temperature. It depends on the temperature, conditions, and material of the surface of the measured body. As the temperature rises, emissivity increases; for metal surfaces, it varies widely depending on the degree of surface cleanliness and the degree of its oxidation. The emissivity coefficient can take values in the range between 0 and 1 [7]. Practically, sensors are calibrated using emitter surfaces that achieve emissivity values of up to 0.99 in the required wavelength range. Bodies with the same temperature but different emissivity values emit different amounts of radiated energy and create their own electrical signals on the detector. There are reference tables that display the emissivity values for various materials.

The optical resolution is the ratio of the sighting spot diameter on the investigated object surface to the distance to the object. When there is a rise in the distance between the infrared sensor and the object surface, the illuminated spot diameter by the investigating sensor expands. This is most clearly shown in Fig. 3.

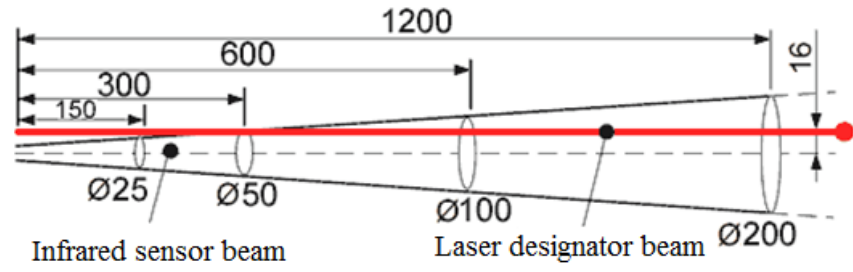

Dimensions in $\mathrm{mm}$

Fig. 3. Pyrometer optical resolution 
Consequently, the measurement of the temperatures of the small objects at large distances is followed by massive observational errors; the reason is that the object surface begins to fall into the spot area, the temperature of which can have a significant difference in comparison with the temperature of the surface of the measured body.

In order to increase the measurement accuracy, it is necessary to maximize the optical resolution of the infrared sensor. This is accomplished by special optical devices, objectives, which reduce the angle of divergence [8].

\section{OVERVIEW}

In the modern market, there is a wide range of automation systems for contactless temperature control of large crowds of people in public places such as airports, the demand for which has increased significantly with the outbreak of the coronavirus infection and the measures taken to prevent its spread in the long run. The ISMTB-ZS-315 thermal imaging complex is considered as an example in this article.

Thermal imaging complex ISMTB-ZS-315 is designed for non-contact temperature measurement of a person or people in a stream. The thermal imager, which is the basis of the operation of this system, allows quick and efficient identification of people who presumably have an elevated body temperature. The complex is used for epidemiological control purposes; along with a thermal imager, the system is equipped with a video camera with an automatic face recognition system.

An example of the obtained images of a video camera and a thermal imager, where the faces of people in the stream and the corresponding body temperatures are determined, is presented in Fig. 4.

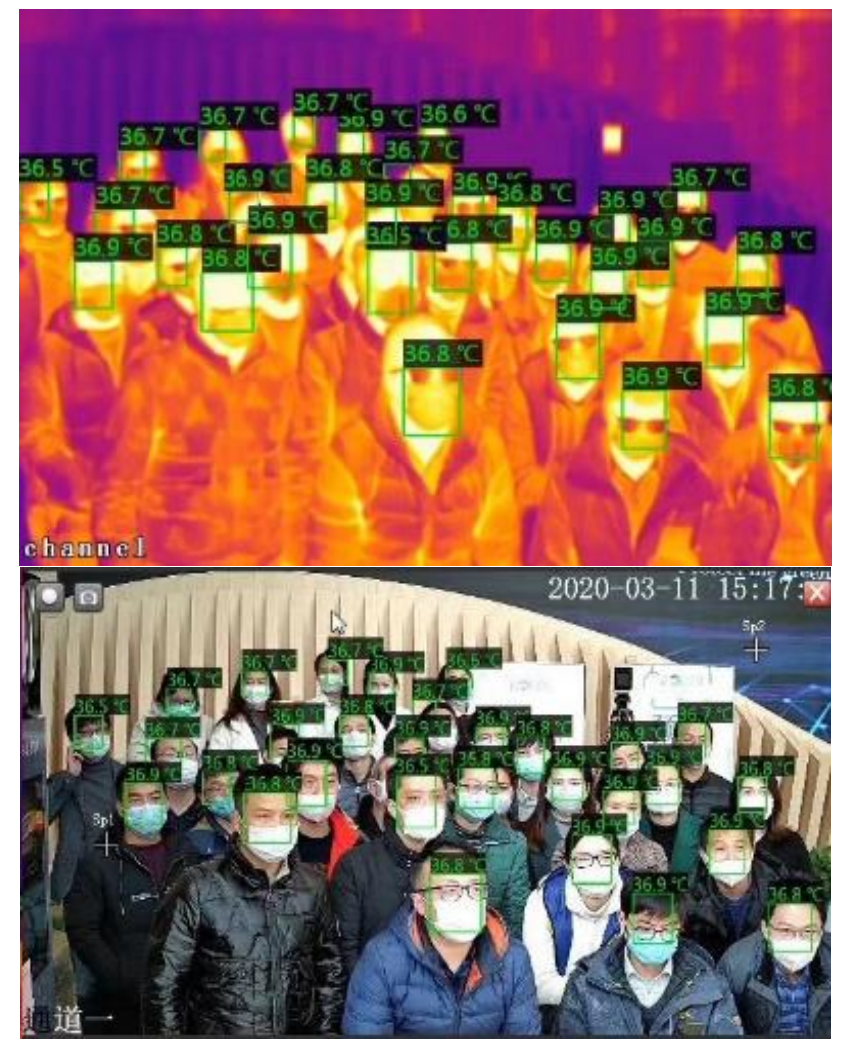

Fig. 4. Images obtained by thermal imaging complex ISMTB-ZS-315.
The capabilities of the mentioned technical solution include the following functions:

- constant temperature control of up to 30 people simultaneously;

- quick identification of people experiencing fever among other people in the stream.

Thermal imaging complex ISMTB-ZS-315 has the following advantages:

- ability of automatic detection of people with elevated body temperature along with the presence of an alarm sound signaling when potentially infected people are detected;

- accuracy of temperature measurements is approximately $\pm 0,3{ }^{\circ} \mathrm{C}$;

- $\quad$ wide field of view and the ability to simultaneously track about 30 people, which is optimal for controlling passenger traffic at entrance lobbies, train stations, airports, subways, and other crowded places;

- face recognition with an intelligent algorithm for matching thermal images and images of the visible wave range;

- $\quad$ sufficiently accurate calibration by a black body;

- $\quad$ simple user interface;

- compact size and easy installation.

The thermal imaging complex consists of three main blocks.

The first part is the optoelectronic unit. It includes a television camera that combines the functions of both a thermal and a video camera. The video camera identifies a feverous person via an intelligent face recognition system; furthermore, it takes a photo and video of a person with an elevated temperature.

The second part of the complex is a black body. It is used to calibrate the thermal imager in order to obtain the most accurate readings by eliminating as many observational errors as possible. It is always in continuous operation, set at $35^{\circ} \mathrm{C}$, and used as a reference for the infrared sensor.

The third part is software that is installed on the computer or laptop of the employee who is responsible for the temperature control. The software allows offline identification of people with an elevated body temperature; it emits a siren sound when a supposedly infected person is detected, whereas it starts recording them [9].

\section{METHODS}

Despite all the previously listed advantages of the ISMTB-ZS-315 thermal imaging complex, which is actively utilized at the airports of the Russian Federation at present, the market price of this complex reaches one million Russian rubles, which is equivalent to about 12,000 euros, making it impossible for its widespread use in other public places such as educational institutions, shopping centers, and hospitals. Therefore, this article proposes to develop a new technical solution that has less broad functionality but at the same time allows much wider use due to maintaining a lower cost.

Based on the review of systems of automatic continuous remote temperature control in flows of people, their main elements can be distinguished as the following components: 
- an infrared sensor that directly detects infrared radiation and performs its processing and reduction to the numerical value of the corresponding temperature value;

- a video camera that registers visible radiation for photographing and filming a specific person who has a high temperature;

- a microcontroller that combines the above peripheral devices into one microprocessor system that performs additional data processing and transmission to other devices via physical communication lines.

In order to solve the given task, it is expedient to use the most widespread modern general-purpose microcontrollers based on the ARM7 / ARM9 microprocessor core, which has the architecture with a reduced instruction set by ARM Ltd.

In Russia, the license for the ARM Cortex-M3 processor core was purchased by ZAO PKK Milandr, located in the city of Zelenograd, in 2008. The Milandr company has developed high-performance 32-bit microcontrollers of the 1986BE9x series for industrial use; this series is produced in various modifications, differing in the number of pins and some functions.

As far as non-Russian manufacturers are concerned, firstly, it is vitally important to mention the STMicroelectronics company. STMicroelectronics has a large share of its own products in the market of electrical radioelements, which includes a wide range of its own microcontrollers. This company has released the STM32 family of microcontrollers, which is based on the ARM Cortex-M3 microprocessor core; these microcontrollers are considered as the benchmark in terms of the balance of performance and cost. These controllers are initially focused on applications with low power consumption and demanding real-time control characteristics and are widely used around the world [10].

In order to develop a system of continuous non-contact temperature control in the flow of people, it is most optimal to use STM32 microcontrollers due to their high availability in the market. However, the further transition to Russian manufacturer's microcontrollers is implied.

Regarding the infrared sensor, it is reasonable to move away from using a thermal imager, which has a high cost and requires a high processor clock frequency to maintain correct and fast operation. Instead, the developing system may include an infrared sensor that registers only one temperature value corresponding to the point or, to be precise, the spot directed by the optical system of the sensor. Therefore, the optical system spot must cover the area that all faces of people of the stream pass through. Additionally, the entire flow of people, which is subject to temperature control, should be lined up in one column, which in turn will reduce the throughput in a certain way, but this will not be as critical as in places of large crowds such as international airports; the proposed system should work quickly enough, for instance, in educational institutions or shopping centers.

The infrared temperature sensor MLX90614 that is produced by Belgium manufacturer Melexis can be considered a valid option for such purposes; its appearance is given in Fig. 5.

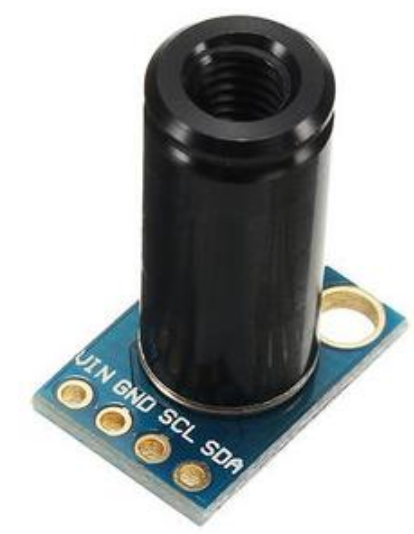

Fig. 5. Infrared temperature sensor MLX90614.

Furthermore, it is advisable to use a continuously operating ultrasonic distance sensor such as HC-SR04, which determines the approach of the next person; the sensor acquired information can serve as a signal to exit the standby mode of the video camera and infrared sensor to reduce their power consumption. The appearance of the $\mathrm{HC}$ SR04 sensor is presented in Fig. 6.

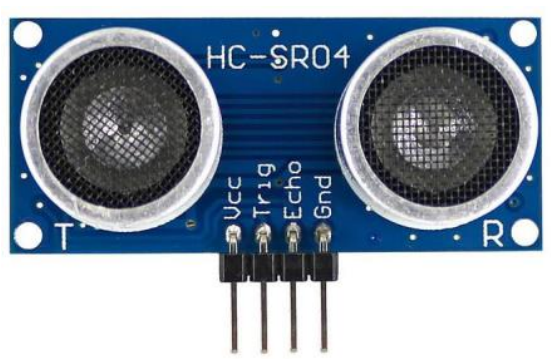

Fig. 6. Ultrasonic distance sensor HC-SR04l.

A video camera is a device for recording visible radiation. It has an optimal optical resolution for the further identification of detected feverous people, and it connects directly to the microcontroller through serial interfaces such as USART.

\section{RESULTS AND DISCUSSION}

Thus, on the basis of the study, for the further development of the proposed technical solution, it is necessary to carry out further research and design work, affecting the following aspects:

1) Selection of the optimal location and direction for the infrared sensor in order to record the temperature of each person from the stream correctly.

2) Minimization of the recorded temperature measurement error by choosing the optimal distance from the sensor to the intended control point and the possible additional improvement of the optical system to minimize the angle of divergence.

3) Determination of the maximum throughput per unit of time and the implementation of additional measures to increase it if necessary.

4) Choosing a specific model of the STM32 microcontroller and writing the program code in the $\mathrm{C}$ language for the correct operation of the microcontroller itself and the peripherals connected to it. 
5) Construction of an electrical schematic diagram, development, routing, and manufacturing of the necessary printed circuit boards (PCB), selection of a case, assembly of the entire system.

6) Development of computer software to provide communication between the developed microprocessor system and a computer, as well as for the convenient display of processed data on the screen.

\section{ACKNOWLEDGMENT}

The author acknowledges with gratitude Radik Muratov, a postgraduate's degree student and a teacher of the Department of Design and Production Technology of Electronic Devices, Institute of Radioelectronics, Photonics and Digital Technologies, Kazan National Research Technical University named after A.N. Tupolev - KAI, Kazan, Russia, for his help in determining the direction of research and development.

\section{REFERENCES}

[1] I. S. Prokopenko, Coronavirus. Killer virus, Moscow: Eksmo Publ., 2020 , p. 224.

[2] K. Chrzanowski, Z. Bielecki, M. Szulim, Comparison of temperature resolution of single-band, dual-band, and multi-band infrared systems, Applied Optics, 1999, vol. 38, no.13. pp. 2820-2823.

[3] W. Thomson, On the thermoelastic, thermomagnetic and pyroelectric properties of matter, Philosophical Magazine, 1878, series 5, vol. 5, pp. 4-26.

[4] L. Boltzmann, Derivation of Stefan's little law concerning the dependence of thermal radiation on the temperature of the electromagnetic theory of light, Annalen der Physik und Chemie, 1884, vol. 258, no.6. pp. 291-294.

[5] Moskowitz, L. Sanford, Advanced Materials Innovation: Managing Global Technology in the 21st century, John Wiley \& Sons Publ., 2018, pp. 165-167.

[6] A. M. Prokhorov, Physics. Great Encyclopedic Dictionary, Moscow: Great Russian Encyclopedia Publ., 1998, p. 944.

[7] G. G. Rabinovich, P.M. Ryabykh, P.A. Khokhryakov, Calculations of the main processes and apparatuses for oil refining. Directory, Moscow: Khimiya Publ., 1979, p. 568.

[8] L. V. Tarasov, Physics of processes incoherent optical radiation generators, Moscow: Radio i svyaz Publ., 1981, p. 440.

[9] ISMTB-ZS-315 - thermal imaging complex for non-contact temperature measurement of people [Online]. Available at: https://skomplekt.com/tovar/1/13/10001360018 (accessed 25 August 2021).

[10] I. N. Ogorodnikov, Microprocessor Engineering: An Introduction to the Cortex-M3, Yekaterinburg: Izdatelstvo Uralskogo universiteta Publ. 2015, p. 116.

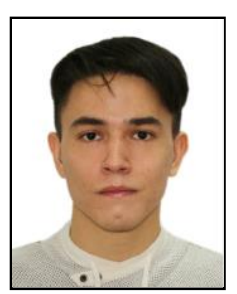

Ilgiz Bakiev was born in the city of Nizhnevartovsk, Russia, on September 29, 1998. He is a Master's degree student at the Department of Design and Production Technology of Electronic Devices, Institute of Radioelectronics, Photonics and Digital Technologies, Kazan National Research Technical University named after A.N. Tupolev - KAI, Kazan, Russia. His field of study is Design and Technology of Electronic Devices.

$\mathrm{He}$ is an R\&D engineer at the Department of Microprocessors and Mechatronics at STC Zarnitza, Kazan, Russia. His research interests are related to AVR and ARM microprocessors programming and their implementation in various electronic devices. 